\title{
Evolutionary and functional characterization of leucoanthocyanidin reductases from Camellia sinensis
}

\author{
Peiqiang Wang ${ }^{1} \cdot$ Lingjie Zhang $^{2} \cdot$ Xiaolan Jiang $^{1} \cdot$ Xinlong Dai $^{1} \cdot$ Lijuan Xu $^{1}$ • \\ Tong $\mathrm{Li}^{1} \cdot$ Dawei Xing ${ }^{1}$ Yanzhi $\mathrm{Li}^{1} \cdot$ Mingzhuo $\mathrm{Li}^{1} \cdot \operatorname{Liping~} \mathrm{Gao}^{2} \cdot \mathrm{Tao} \mathrm{Xia}^{1}$
}

Received: 28 April 2017 / Accepted: 2 September 2017 / Published online: 8 September 2017

(C) The Author(s) 2017. This article is an open access publication

\begin{abstract}
Main conclusion LARs promoted the biosynthesis of catechin monomers and inhibited their polymerization. The accumulation of catechin monomers and polymers was increased by up-regulating the expression of NtLAR and NtANRs in CsMYB5b transgenic tobacco.
\end{abstract}

Tea is rich in polyphenolic compounds, and catechins are the major polyphenols in tea. The biosynthesis of polyphenols is closely related to the expression of the leucoanthocyanidin reductase $(L A R)$ and anthocyanidin reductase (ANR) genes. In this paper, an evolutionary analysis and functional characterization of three CsLARs were performed. The phylogenetic tree showed that plant LARs could be grouped into three, including gymnosperms, monocotyledons and dicotyledons (clusters I and II). The eighth amino acid residue in a conserved LAR-specific motif is changeable due to a transversion $(\mathrm{G} \rightarrow \mathrm{T})$ and transition $(\mathrm{G} \rightarrow \mathrm{C})$ that occur in the corresponding codon. Therefore, plant LARs can be classified as G-type, A-type and S-type LARs due to this variable amino acid residue. Although $(2 \mathrm{R}, 3 \mathrm{~S})$-trans-flavan3 -ols were the products of recombinant CsLARs proteins

Electronic supplementary material The online version of this article (doi:10.1007/s00425-017-2771-z) contains supplementary material, which is available to authorized users.

Liping Gao

gaolp@ahau.edu.cn; gaolp62@126.com

Tao Xia

xiatao62@ahau.edu.cn; xiatao62@126.com

1 State Key Laboratory of Tea Plant Biology and Utilization, Anhui Agricultural University, Hefei 230036, Anhui, China

2 School of Life Science, Anhui Agricultural University, 130 West Changjiang Rd, Hefei 230036, Anhui, China expressed in Escherichia coli, both (2R, 3S)-trans and (2R, 3R)-cis-flavan-3-ols were detected in tobacco overexpressing CsLARs. However, a butanol/HCl hydrolysis assay indicated that overexpression of the CsLARs caused a decrease in polymerized catechins. A hybridization experiment with CsLARc + AtPAP1 also showed that no polymers other than epicatechin, catechin and glycoside were detected, although the accumulation of anthocyanins was markedly decreased. CsMYB5b promoted the biosynthesis of both flavan-3-ols and proanthocyanidins (PAs). Therefore, LARs promoted the biosynthesis of catechin monomers and inhibited their polymerization. The accumulation of catechin monomers and polymers was increased by up-regulating the expression of the NtLAR and NtANRs in CsMYB5b transgenic tobacco.

Keywords Catechins - Leucoanthocyanidin reductases . Phylogenetic analysis · Tea plant
Abbreviations
ANR Anthocyanidin reductase
C Catechin
DFR Dihydroflavonol 4-reductase
DMACA Dimethylaminocinnamaldehyde
EC Epicatechin
GC Gallocatechin
LAR Leucoanthocyanidin reductase

\section{Introduction}

Polyphenolic compounds are a large class of plant secondary metabolites that are ubiquitously present in many plants, and they mainly consist of flavones, flavonols, anthocyanins, isoflavones, condensed tannins (CT; or PAs) and other derivatives (Winkel-Shirley 2001; Tanner et al. 2003; Xie 
et al. 2003). Different classes of polyphenolic compounds contribute in various ways to plant growth and defense against biotic and abiotic stress, including pathogen infections, ultraviolet radiation and herbivory (Dixon and Sumner 2003; Mellway et al. 2009). Additionally, certain polyphenolic compounds can act as antioxidants and protect humans from cardiovascular disease, diabetes, ultraviolet radiation and obesity (Cos et al. 2004; Nichols and Katiyar 2010; Wang et al. 2013). Flavan-3-ols and PAs greatly contribute to the astringency of tea, wine and certain fruits, such as persimmon (Diospyros kaki) (Kennedy et al. 2008; Ma et al. 2010; Liu et al. 2012).

Tea, which is a perennial evergreen woody plant, is rich in polyphenolic compounds. In tea, dissociative flavan-3-ol monomers are mainly distributed in the leaves of the aerial parts of the plant, and polymeric catechins are distributed in the stems and root (Jiang et al. 2013, 2015). Dissociative flavon-3-ols are also called catechins and include (2R, 3S)trans-flavan-3-ols (catechin, C, and gallocatechin, GC) and (2R, 3R)-cis-flavan-3-ols (epicatechin, EC; epigallocatechin, EGC; epicatechin gallate, ECG; and epigallocatechin gallate, EGCG) (Qian et al. 2015). Catechins play a crucial role in the dominating astringent taste of tea and possess a number of effects that are beneficial to human health, particularly the galloylated catechins (Chung et al. 2003; Cui et al. 2016).

The biosynthesis pathway (Fig. 1) of polyphenolic compounds has been characterized via genetic and biochemical approaches in many plant species, including Arabidopsis thaliana, Vitis vinifera, Populus trichocarpa, Theobroma cacao and Camellia sinensis (Nesi et al. 2001; Bogs et al. 2007; Li et al. 2012; Liu et al. 2013; Pang et al. 2013). Most structural genes in the flavonoid metabolism pathway have been identified. ANRs catalyze anthocyanins to form epicatechins (epiafzelechin, EFZ; EC; and EGC), and LARs convert the leucoanthocyanidins into the corresponding catechins (afzelechin, AFZ; C; and GC) in vitro (Stafford 1991; Tanner et al. 2003). The biosynthetic pathway of galloylated catechins was shown to be a two-step enzyme reaction in the tea plant (Liu et al. 2012). The functions of two ANRs and one LAR from the tea plant have been identified in previously published papers, but some questions remain (Pang et al. 2013).

Because LARs are essential reductases in the flavonoid pathway, studies have been conducted to investigate LAR enzymes since the 1980s. Stafford and Lester $(1982,1984)$ first detected the enzyme activity of converting $(+)$-dihydroquercetin (DHQ) to leucocyanidin and then to $(+)$-catechin with NADPH as the H-donor in crude protein extracts derived from Pseudotsuga menziesii. DuLAR, which was the first LAR protein purified from the PA-rich leaves of Desmodium uncinatum, was confirmed to synthesize C, AFZ and GC with 3,4-cis-leucoanthocyanidins as substrates by expressing recombinant protein in Escherichia coli (E. coli), tobacco (Nicotiana tabacum) and white clover (Trifolium repens) (Tanner et al. 2003). Many LAR genes have been identified in various plants, such as $V$. vinifera, Medicago truncatula and Ceratocystis polonica (Kristiansen 1986; Bogs et al. 2005; Hammerbacher et al. 2014). Subsequently, the crystal structure and catalytic mechanism of VvLAR in vitro were described in detail, which helped us to further understand the function of LARs (Mauge et al. 2010).

Recently, a new discovery in a research study investigating the role of LARs in PA synthesis reported that MtLAR could convert $4 \beta$-( $S$-cysteinyl)-epicatechin, a new substrate, into $\mathrm{EC}$ in vitro. $4 \beta$-( $S$-cysteinyl)-epicatechin, which is also a carbocation form, played an important role in non-enzymatic polymerization and served as a PA extension unit (Liu et al. $2016)$. However, the source of $4 \beta$-( $S$-cysteinyl)-epicatechin is currently unknown.

In this paper, three CsLARs in tea plants were cloned and functionally characterized in vivo and in vitro. In addition, the evolutionary relationship between the CsLARs and other plant LARs from gymnosperms and angiosperms is discussed. All of the data suggest that CsLARs are responsible for the biosynthesis of both (2R, 3S)-trans- and (2R, 3R)-cis-flavan-3-ols in the trangenetic CsLARs tobacco. Coexpression of the $A N R$ and $L A R$ genes promotes the biosynthesis of both flavan-3-ols and PAs.

\section{Materials and methods}

\section{Materials}

The tea plants (C. sinensis cv. Shuchazao) used in this experiment were grown in an experimental tea field at Anhui Agricultural University, Hefei, China (East longitude 117.27, North latitude 31.86). Leaves at different developmental stages (buds and 1st, 2nd, 3rd and 4th leaves), mature leaves, tender stems and roots were harvested and immediately frozen in liquid nitrogen for further study. The wild-type Arabidopsis thaliana used in our laboratory was ecotype Columbia 0 (Col-0), which was grown in a chamber at a constant temperature of $16 \pm 3{ }^{\circ} \mathrm{C}$ and a $16 / 8 \mathrm{~h}$ (light/dark) photoperiod. The tobacco (Nicotiana tabacum cv. G28) used for the transgenic assays was provided by the University of Science and Technology of China (Hefei, Anhui, China) and grown in a growth chamber at a constant temperature of $24 \pm 3{ }^{\circ} \mathrm{C}$ and a $12 / 12 \mathrm{~h}$ (light/dark) photoperiod.

Escherichia coli DH5 $\alpha$ and BL21 (DE3) (TransGen Biotech, Beijing, China) were used as the host strain and expression strain for the prokaryotic expression, respectively. Agrobacterium tumefaciens $\mathrm{C} 58 \mathrm{C} 1$ and EHA105 were kindly provided by the University of Science and Technology of China. 
Fig. 1 Schematic representation of the biosynthetic pathway of flavan-3-ols

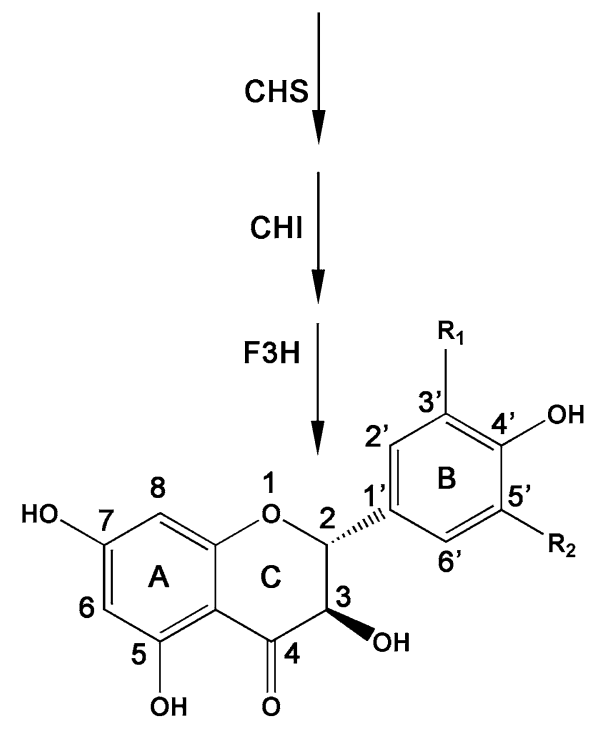

2R,3R-dihydroflavonols

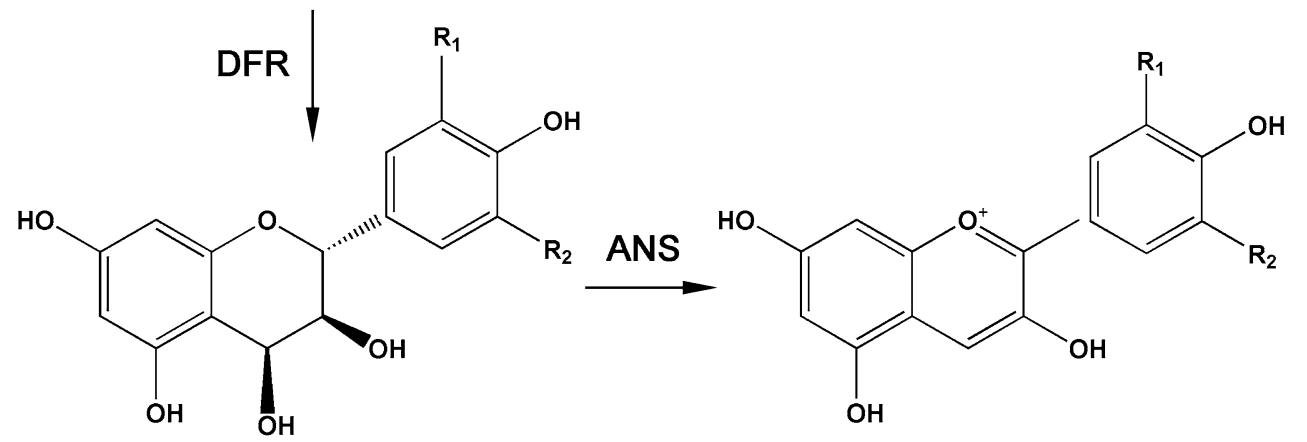

2R,3S,4S-flavan-3,4-diols

(Leucoanthocyanidins)

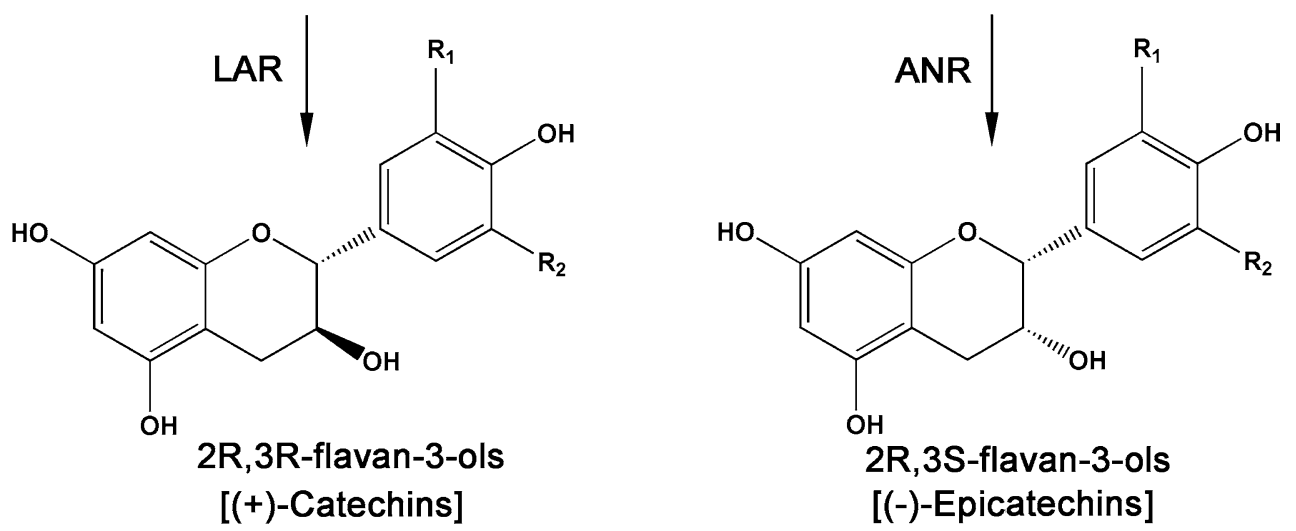

The substrates DHQ, dihydromyricetin (DHM) and dihydrokaempferol (DHK) and standard C, GC, EC, EGC, ECG, EGCG, procyanidin B2 and cyanidin-3-O-glucosides were purchased from Sigma (St. Louis, MO, USA).

\section{Cloning of CsLARa, CsLARb, and CsLARc}

Total RNA was extracted from the aforementioned organs of the tea plants using RNAiso mate and RNAiso Plus (Takara, 
DaLian, China) according to the manufacturer's instructions. The reverse transcription reaction was carried out using the PrimeScript RT Reagent Kit (Takara). In addition, the $3^{\prime}$ and 5' RACE (rapid amplification of cDNA ends) Kit (Clontech, Mountain View, CA, USA) was used for the RACE library construction following the manufacturer's instructions. The full open reading frame (ORF) was amplified by high-fidelity PCR using cDNA from the total RNA. The ORF primers of $L A R s$ are listed in Suppl. Table S1. The PCR procedure was performed at $98^{\circ} \mathrm{C}$ for $30 \mathrm{~s}, 30$ cycles at $98^{\circ} \mathrm{C}$ for $10 \mathrm{~s}$, $60{ }^{\circ} \mathrm{C}$ for $30 \mathrm{~s}$ and $72{ }^{\circ} \mathrm{C}$ for $45 \mathrm{~s}$, followed by a final extension at $72{ }^{\circ} \mathrm{C}$ for $10 \mathrm{~min}$. The PCR amplification products were gel purified using a Gel Extraction Kit (Aidlab, Beijing, China), ligated into an Easy-Blunt vector (TransGen Biotech) and then transformed into DH5 $\alpha$ competent cells for sequencing.

\section{Expression of CsLARs in $E$. coli and their functional validation}

A double gene expression strategy was performed for the functional validation of the CsLARs. The ORFs of CsLARs and $C s D F R a$ were cloned into the expression vector pRSFduet (Novagen, Carlsbad, CA, USA), which contains two multiple cloning sites (MCS I and MCS II). The primers used for $C s L A R s$ and $C s D F R a$ to construct the protein expression vectors are listed in Suppl. Table S1. The PCR procedure was performed at $98{ }^{\circ} \mathrm{C}$ for $30 \mathrm{~s}$, followed by 30 cycles of $98{ }^{\circ} \mathrm{C}$ for $10 \mathrm{~s}, 60{ }^{\circ} \mathrm{C}$ for $30 \mathrm{~s}$ and $72{ }^{\circ} \mathrm{C}$ for $45 \mathrm{~s}$, with a final extension at $72{ }^{\circ} \mathrm{C}$ for $10 \mathrm{~min}$. The PCR products and empty pRSFduet vector were digested with the corresponding restriction enzymes (New England Biolabs) at $37{ }^{\circ} \mathrm{C}$ for $12 \mathrm{~h}$. After gel purification, the products were ligated into the digested vector using T4 ligase (New England Biolabs) at $16^{\circ} \mathrm{C}$ for $12 \mathrm{~h}$ before being transformed into DH $5 \alpha$ competent cells for sequencing. After sequencing, the empty vector (pRSFduet), pRSFduet-CsDFRa, pRSFduet-CsDFRa + CsLARa, pRSF-CsDFRa + CsLARb and pRSF-CsDFR + CsLARc were transformed into expression strain BL21 (DE3) for protein expression. A single colony confirmed by PCR was inoculated into Luria-Bertani (LB) broth containing $50 \mathrm{mg} / \mathrm{L}$ kanamycin at $37{ }^{\circ} \mathrm{C}$. When the $\mathrm{OD}_{600}$ reached 0.6-0.8, IPTG (isopropyl $\beta$-D-1thiogalactopyranoside) was added to a final concentration of $1 \mathrm{mM}$ to induce protein expression. After the cell cultures were incubated at $28^{\circ} \mathrm{C}$ for $6-8 \mathrm{~h}$, dihydroflavonols (DHK, DHQ and DHM), which could be used as substrates by CsDFRa and converted into leucoanthocyanidins, were added to a final concentration of $100 \mu \mathrm{M}$. After $8 \mathrm{~h}$, the supernatant of each bacterial suspension was harvested by centrifugation ( $3500 \mathrm{~g}$ for $15 \mathrm{~min}$ at $4{ }^{\circ} \mathrm{C}$ ) and extracted three times with ethyl acetate. The merged supernatant was concentrated to remove ethyl acetate at a low temperature. Finally, the products were dissolved in $200 \mu \mathrm{L}$ methanol. To identify the products of DFRa + LARs, reverse-phase HPLC (Shimadzu, Kyoto, Japan) and ultrahigh-performance liquid chromatography coupled to a mass spectrometer (UPLC-MS/MS) (Agilent, Santa Clara, CA, USA) were used as previously described (Cui et al. 2016).

\section{Agrobacterium-mediated transformation of Arabidopsis} and tobacco

The ORFs of the CsLARs were cloned into the Gateway Entry vector pDONR207 using the Gateway BP Enzyme mix according to the manufacturer's instructions (Invitrogen, Carlsbad, CA, USA). The primers are listed in Suppl. Table S1. After sequencing, the entry vectors were recombined into the plant transformation destination vector pCB2004 using the LR Enzyme mix (Invitrogen). The recombinant pCB2004-LARs were electroporated into Agrobacterium tumefaciens EHA105 and C58C1. A single EHA105 colony containing the target construct was confirmed by PCR and inoculated into liquid LB medium containing $50 \mathrm{mg} / \mathrm{L}$ kanamycin and $50 \mathrm{mg} / \mathrm{L}$ spectinomycin at $28{ }^{\circ} \mathrm{C}$ until the $\mathrm{OD}_{600}$ of the cell suspension reached 0.6. The Agrobacterium cells were collected by centrifugation (3500, $15 \mathrm{~min}$ ) and used for gene transformation in tobacco following a previously reported method (Fisher and Guiltinan 1995; Wang et al. 2014). Similarly, an Agrobacterium tumefaciens $\mathrm{C} 58 \mathrm{C} 1$ colony was transferred into Arabidopsis using the floral-dip method as previously described (Clough and Bent 1998; Cai et al. 2014).

\section{Hybridization of different transgenic tobacco lines}

Well-grown T1 generation plants of CsLARs transgenic tobacco and AtPAP1 transgenic tobacco were chosen, and their stamens were gently removed before pollination. The pollen of tobacco overexpressing CsLARs and AtPAP1 was then applied to the pistils of AtPAP1 and CsLARs transgenic tobacco, respectively. The pollinated pistils were protected by a transparent bag until the seeds were ripe. The newly grown seedlings were used in subsequent experiments after PCR verification.

\section{Extraction and quantification of catechins, anthocyanins and PAs}

To analyze the catechins in the tea plants, $0.2 \mathrm{~g}$ of fresh samples (buds, tender leaves, young stem and young roots) were ground in liquid nitrogen and extracted with an extraction solution ( $80 \%$ methanol and $20 \%$ water), followed by vortexing and sonicating for $30 \mathrm{~min}$ at a low temperature. Then, the samples were centrifuged at $3500 \mathrm{~g}$ for $15 \mathrm{~min}$, and the residues were re-extracted twice as described above 
until the final volume of the pooled supernatants was $2 \mathrm{~mL}$. The supernatants were then extracted three times with chloroform and three times with ethyl acetate. The pooled supernatant was concentrated to remove the ethyl acetate at a low temperature with a vacuum pump. Finally, the product was dissolved in $200 \mu \mathrm{L}$ methanol for further analysis. Six main catechins were quantitatively analyzed following a previously described HPLC method with corresponding standards (Jiang et al. 2013).

To analyze the anthocyanins in the transgenic tobacco flowers, $0.5 \mathrm{~g}$ of fresh petals was ground in liquid nitrogen and extracted with an extraction solution (80\% methanol, $19.9 \%$ water and $0.1 \%$ hydrochloric acid, by vol.), followed by vortexing and sonicating for $30 \mathrm{~min}$ at a low temperature. Then, the samples were centrifuged at $3500 \mathrm{~g}$ for $15 \mathrm{~min}$, and the residues were re-extracted twice as described above until the final volume of the pooled supernatants was $2 \mathrm{~mL}$. The absorption of the supernatants was measured at $525 \mathrm{~nm}$.

To analyze the PAs in the transgenic tobacco flowers, $0.5 \mathrm{~g}$ of fresh petals was ground in liquid nitrogen and extracted with an extraction solution (70\% acetone, 29.5\% water, and $0.5 \%$ acetic acid), followed by vortexing and sonicating for $30 \mathrm{~min}$ at a low temperature. Then, the samples were centrifuged at $3500 \mathrm{~g}$ for $15 \mathrm{~min}$, and the residues were re-extracted twice as described above. The pooled supernatants were extracted three times with chloroform and three times with ethyl acetate. The pooled supernatant was steamed to remove the ethyl acetate at a low temperature using a vacuum pump. Finally, the product was dissolved in $200 \mu \mathrm{L}$ methanol for further analysis.

To detect the soluble PAs in the tobacco flowers, the dimethylaminocinnamaldehyde (DMACA) reagent $(0.2 \%$ DMACA, w/v) was used as previously described ( $\mathrm{Li}$ et al. 1996). The absorption of the reaction mixture (DMACA: supernatants $=10: 3$ ) was measured at $640 \mathrm{~nm}$. To further identify the product, UPLC coupled to a triple quadrupole mass spectrometer (QQQ-MS) (Agilent) was used following a previously described method (Jiang et al. 2015). A quantitative established method based on the multiple reaction monitoring (MRM) mode of the UPLC-QQQ-MS was used to quantify the products in the different transgenic lines, with EC, C and procyanidin B2 as the standards (Wu et al. 2016).

To extract and detect the soluble PAs from the Arabidopsis seeds, the same method was applied, but $50 \mathrm{mg}$ of dry seeds was used as the grinding material. To quantify the total soluble PAs, the DMACA reagent (0.2\% DMACA, w/v) was applied using procyanidin $\mathrm{B} 2$ as the standard.

To determine the insoluble PAs in the tobacco flowers and seeds from Arabidopsis, the residues were dried using a vacuum drier. Then, $1 \mathrm{~mL}$ of the butanol- $\mathrm{HCl}$ reagent was added to the dried residues, and the mixture was sonicated for $30 \mathrm{~min}$ at room temperature, followed by centrifugation at $3500 \mathrm{~g}$ for $15 \mathrm{~min}$. The absorption (A1) of the supernatants was measured at $525 \mathrm{~nm}$ to determine the amount of background absorption. After boiling the butanol- $\mathrm{HCl}$ reagent and residue mixtures for $1 \mathrm{~h}$ at $95^{\circ} \mathrm{C}$, the absorbance (A2) at $525 \mathrm{~nm}$ was measured again. The normalized absorbance of the insoluble PAs was calculated by subtracting the A2 values from the $A 1$ values and converting that value into $P A$ equivalents using cyanidin-3-O-glucoside as the standard.

\section{Quantitative real-time PCR}

RNA that was extracted from the various tissues was quantified with a spectrophotometer (NANODROP 2000, Thermo Scientific). Reverse transcription of the RNA into cDNA was performed using $2 \mu \mathrm{L} 5 \times$ PrimeScript RT Master Mix (Takara) and 500 ng RNA in a reaction volume of $10 \mu \mathrm{L}$. In addition, the cDNA was diluted to $25 \%$ (v/v) with deionized water before being used as the template. The quantitative real-time PCR was performed in a reaction mixture volume of $20 \mu \mathrm{L}$ containing $10 \mu \mathrm{L}$ SYBR Green PCR Master Mix (Takara), $1.1 \mu \mathrm{L}$ cDNA and $0.8 \mu \mathrm{L}$ forward and reverse primer $(10 \mu \mathrm{M})$. The PCR cycling parameters used were as follows: $95{ }^{\circ} \mathrm{C}$ for $30 \mathrm{~s}$ and 40 cycles of $95{ }^{\circ} \mathrm{C}$ for $5 \mathrm{~s}, 30 \mathrm{~s}$ at $60{ }^{\circ} \mathrm{C}$ and $30 \mathrm{~s}$ at $72{ }^{\circ} \mathrm{C}$, followed by a melting curve analysis from $55{ }^{\circ} \mathrm{C}$ to $95{ }^{\circ} \mathrm{C}$. The transcription abundance was normalized to the transcription abundance of glyceraldehyde-3-phosphate dehydrogenase (GAPDH) and was calculated from three technical replicates. The relative expression level was calculated by a previously published method (Wang et al. 2014).

\section{Results}

\section{Gene cloning and sequence analysis of the CsLARs}

Using reverse genetic approaches, the function of a CsLAR from the tea cultivar TRI2043 was verified in previous reports (Pang et al. 2013). At least three transcripts of the CsLARs genes were screened against the National Center Biotechnology Information (NCBI, http://www.ncbi.nlm. nih.gov) database by homologous sequence analysis.

In this paper, the full-length cDNA sequences of three CsLARs were obtained from the local cultivar 'Shuchazao' using the RACE technology. The results showed that the full cDNA lengths of the three CsLARs were 1518, 1401 and $1595 \mathrm{bp}$ with 1029,984 and $1197 \mathrm{bp}$ open reading frames (ORFs), respectively. Their translated proteins were 37, 36 and $43 \mathrm{kDa}$, and the predicted $\mathrm{pI}$ values were $5.43,5.24$ and 5.27, respectively. The three CsLARs genes were named $C s L A R a, C s L A R b$ and $C s L A R c$ and uploaded to NCBI (GenBank accession numbers are KY615698, KY615700 and KY615699, respectively). 
The amino acid sequences of the CsLARs were aligned with LAR proteins in other species using the DNAMAN program (Lynnon Corporation, San Ramon, CA, USA). The results showed that CsLARa had 71.88 and $54.41 \%$ amino acid sequence identity with CsLARb and CsLARc, respectively. The amino acid sequence alignment diagrams (Fig. 2) showed that the CsLARs have a putative glycine-rich NADP-binding domain (marked with a green dot) that is located in the $\mathrm{N}$-terminal region, and several substrate binding sites (marked with pink dot) scattered in different regions were predicted based on the scheme of VvLAR (Mauge et al. 2010). Three LAR-specific amino acid motifs, RFLP, ICCN and THD (marked with a blue box), were consistent with other LARs (Hammerbacher et al. 2014).

\section{Evolutionary analysis of the plant LAR family}

Two hundred and six candidate LAR sequences (containing duplicate sequences) were screened in different species using the CsLARs as seed sequences against the NCBI database with the blastP method (identity $>51 \%$ ). The sequences with less than 50\% similarity to the CsLARs are IFR homologous sequences. To obtain as many LAR sequences of different species as possible, we also searched the transcriptomes of different species with the base sequences of the CsLARs

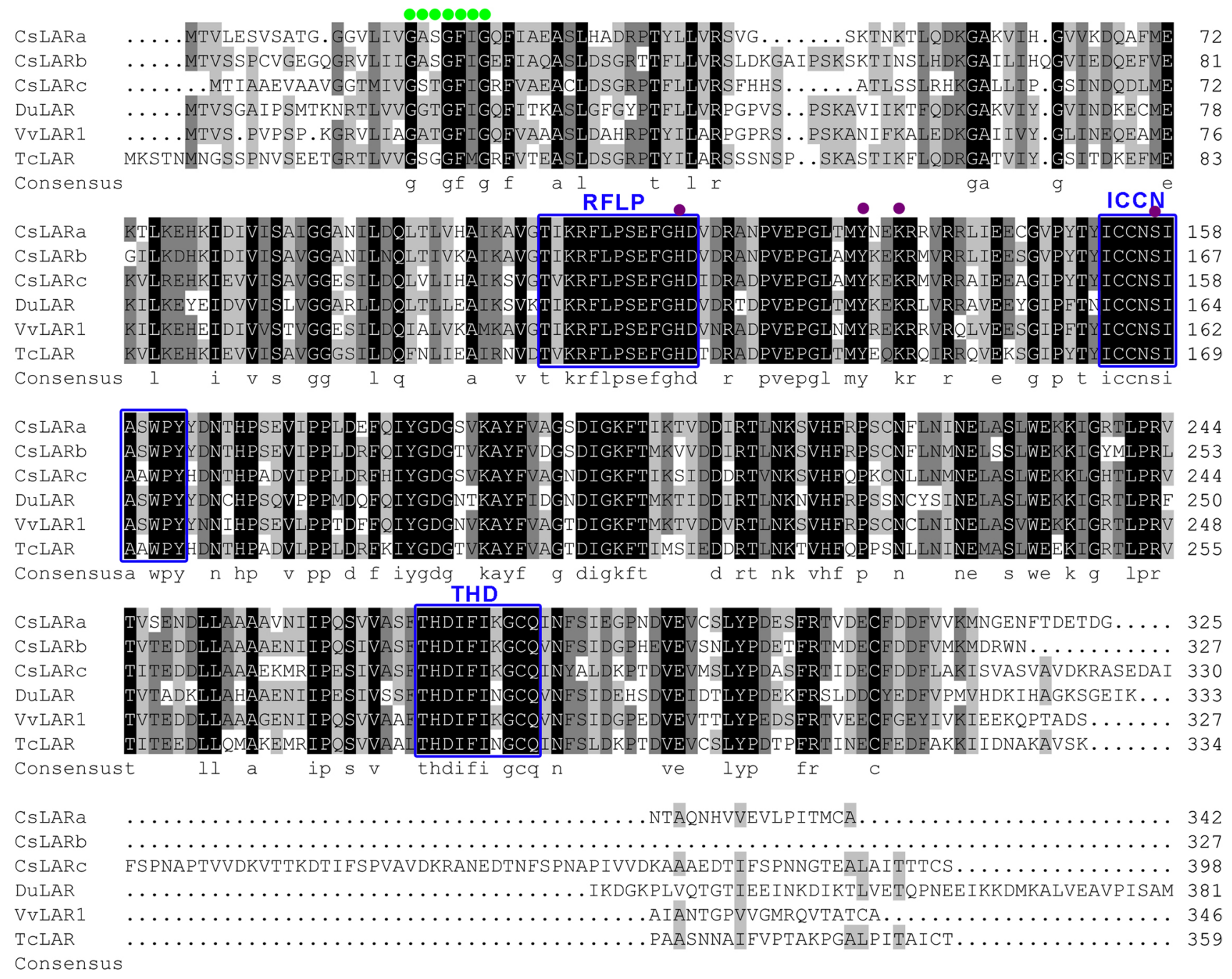

Fig. 2 Sequence comparison of the LARs from the tea plant and other selected species. NADP-binding domain and substrate-binding sites are marked by green dots and pink dots, respectively, according to the scheme of VvLAR. LAR-specific motifs RFLP, ICCN and THD are marked by a blue box. Sequences are from Theobroma cacao (ADD51358), Vitis vinifera (AAZ82410) and Desmodium uncinatum (CAD79341). Identical amino acids are marked by white letters on a black background; conservative amino acids are marked by a dark gray background (similarity $>75 \%$ ); similar amino acids are indicated by black letters on a light gray background (similarity $>50 \%$ ); other amino acids are marked by black letters on a white background (similarity $<50 \%$ ). Sequence alignment was performed using the DNAMAN program 
to find sequences with high similarity (identity $>65 \%$ ). Then, we used the ORF finder software (https://www.ncbi. nlm.nih.gov/orffinder/) to find the open reading frames and translated them into amino acids. The functional LARs reported previously were constructed as models to test the candidate sequences (Suppl. Fig. S1). All LAR sequences have the LAR-specific motifs (RFLP, ICCN, and THD) and
NADPH-binding site GXXGXXG located at the N-terminus. To illustrate this phenomenon more clearly, we chose a subset of these sequences for analysis in this paper.

A phylogenetic tree was constructed using the neighborjoining method based on the above-mentioned amino acid sequences (Fig. 3a). The phylogenetic analysis showed that plant LARs could be grouped into two main clades. One
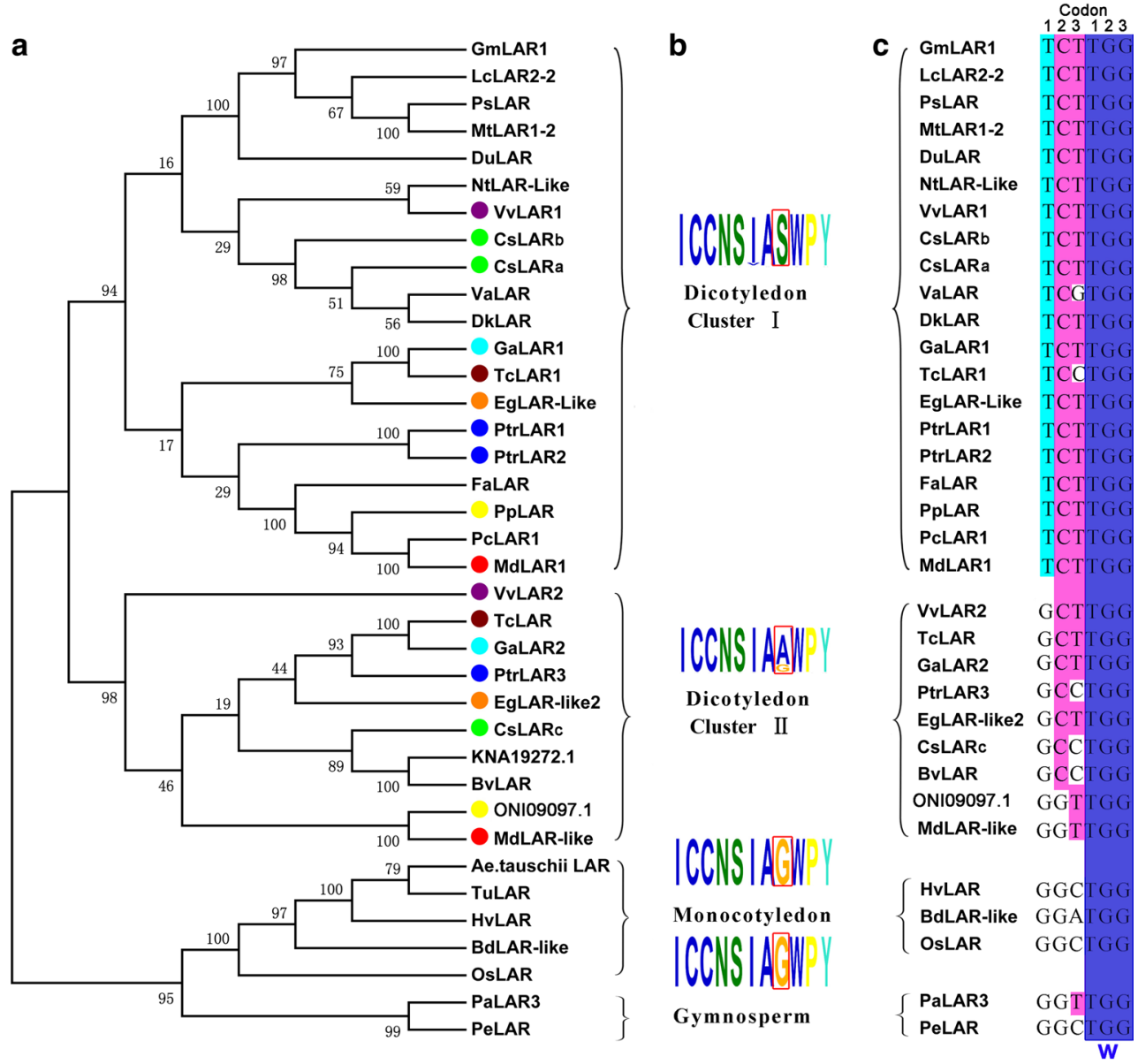

Fig. 3 Phylogenetic relationships of LAR proteins from different species and a conserved motif and the corresponding codon analysis. a The phylogenetic tree of LAR proteins from the tea plant and other species. The phylogenetic analysis was performed using the neighborjoining method with 1000 bootstrap replicates by MEGA version 5.0. The numbers indicate the confidence percentages. The accession numbers of the protein sequences obtained from GenBank are as follows: PaLAR3 (Picea abies, AMB51440.1); PeLAR (Pinus taeda, CAI56321.1); OsLAR (Oryza sativa, CAI56328); HvLAR (Hordeum vulgare, CAI56320); TuLAR (Triticum urartu, EMS45018.1); BdLAR-like (Brachypodium distachyon, XP_003561802.1); Ae.tauschii LAR (Aegilops tauschii, EMT15815.1); CsLARa (Camellia sinensis, KY615698), CsLARb (Camellia sinensis, KY615700), CsLARc (Camellia sinensis, KY615699); GaLAR1 (Gossypium arboretum, CAI56319); GaLAR2 (Gossypium arboretum, CAI56323.1); TcLAR1 (Theobroma cacao, EOY02147.1); TcLAR (Theobroma cacao, ADD51358); PtrLAR1 (Populus tomentosa, EEE89746); PtrLAR2 (Populus tomentosa, EEF01056); PtrLAR3 (Populus trichocarpa, EEF06163); VvLAR1 (Vitis vinifera,
AAZ82410); VvLAR2 (Vitis vinifera, AAZ82411); BvLAR-like (Beta vulgaris, XP_010695625.1); MdLAR1 (Malus domestica, AFZ93007.1); MdLAR-Like (Malus domestica, XP_008356649.1); PpLAR (Prunus persica, EMJ23473.1); EgLAR-Like 2 (Eucalyptus grandis, KCW49054.1); EgLAR-Like (Eucalyptus grandis, XP_010044298.1); DuLAR (Desmodium uncinatum, CAD79341); GmLAR (Glycine max, AEM23933.1); LcLAR2-2 (Lotus corniculatus, ABC71329.1); MtLAR1-2 (Medicago truncatula, AES62081.1); PsLAR (Pisum sativum, AII26024.1); NtLAR-Like (Nicotiana tomentosiformis, XP_009614312.1); PcLAR1 (Pyrus communis, ABB77696.1); VaLAR (Vaccinium ashei, BAM42674.1); DkLAR (Diospyros kaki, ACI41981.1); and FaLAR (Fragaria ananassa, AFP99288.1); KNA19272.1 and ONI09097.1 were two hypothetical proteins selected from Spinacia oleracea and Prunus persica. b Three types of conserved ICCN motifs in LAR amino acid sequences. The amino acid residue in the red box was changeable during the evolutionary process. $\mathbf{c}$ The corresponding codons of the ICCN motif. The blue color indicates the codon of a conserved Trp (W), and the red color indicates the codon of the variable amino acid residue 
clade included the dicotyledon group, and the other included the gymnosperm group and the monocotyledon group, which suggested that monocotyledon LARs were evolutionarily closer to gymnosperm LARs than to dicotyledon LARs.

The dicotyledonous LARs could be further clustered into two subgroups, which were defined as cluster I and cluster II. The LARs in C. sinensis, V. vinifera, T. cacao, P. trichocarpa, Gossypium arboreum, Malus $\times$ domestica, Pyrus communis, Eucalyptus grandis and Prunus persica were clustered into cluster I and cluster II. However, the LARs from Leguminosae, such as Glycine max, Lotus corniculatus, Pisum sativum and M. truncatula, constituted a monophyletic group that belonged to cluster I.

An amino acid residue varied in a conserved LAR-specific amino acid motif (ICCNSIAG/A/SWPY) (Fig. 3b). The sequence alignment of LARs in the phylogenetic tree showed that the eighth amino acid residue in the "ICCNSIAG/A/ SWPY" motif in the gymnosperm and monocotyledon groups was a Gly residue, and Ser and Ala were in the same location in dicotyledonous cluster I and cluster II, respectively (Fig. 3b). Therefore, the gymnosperm and monocotyledon groups and dicotyledonous cluster I and cluster II could be named G-type LARs, S-type LARs and A-type LARs, respectively, based on this variable amino acid. The corresponding nucleotide sequence alignment showed that a transversion $(\mathrm{G} \rightarrow \mathrm{T})$ and a transition $(\mathrm{G} \rightarrow \mathrm{C})$ occurred in the first and second position of this codon, respectively (Fig. 3c). This finding suggested that the plant LAR family might have undergone a mutation in the eighth amino acid position in the conserved motif ICCN due to evolutionary pressures imposed by natural selection. According to the crystal structure of the VvLAR protein, the ICCN motif is located near the substrate binding site, suggesting this mutation may be of importance to the activity of the plant LARs (Mauge et al. 2010).

\section{Correlation between the content of the catechins and the transcription levels of the CsLARs in tea plants}

Tea is rich in polyphenolic compounds, which mainly consist of six flavan-3-ols: EGCG, ECG, EGC, EC, GC and C. The contents of the six main catechins extracted from the bud, leaf, stem and young root of the tea plants were detected by HPLC (Cui et al. 2016). The results showed that the contents of the epicatechins (EGCG, ECG, EGC and EC) were much higher than those of the nonepicatechins (C and GC), and the contents of the galloylated catechins (EGCG and ECG) were much higher than those of the nongalloylated catechins $(\mathrm{C}$, GC, EC, and EGC). EGCG and ECG were the predominant catechins in the leaves (Suppl. Fig. S2a). The accumulation of catechins was greater in the buds and younger leaves than in the mature leaves, stems and roots. However, no other catechins, except EC, were detected in the roots.
To investigate the transcription patterns of the CsLARs in different tea organs, quantitative real-time PCR (qRT-PCR) was performed. The results showed that the transcription profiles of $C s L A R a$ and $C s L A R b$ were similar, exhibiting relatively high levels in the tender shoots and leaves and low levels in the stems and roots (Suppl. Fig. S2b). CsLARC was preferentially expressed in the roots and moderately expressed in the leaves and stems; its expression level in the roots was twice as abundant as in the leaves. The transcription levels of the three CsLARs in the mature leaves were the lowest among all of the leaf organs, which was consistent with the catechin distribution profiles in the mature leaves.

A correlation analysis showed that the accumulation of the catechins was positively correlated with the expression of $C s L A R a$ and $C s L A R b$. The transcriptional levels of $C s L A R a$ and $C s L A R b$ had the highest correlation with the accumulation pattern of EGC and the lowest correlation with the accumulation pattern of $\mathrm{C}$; the corresponding correlation coefficients were 0.7323 and 0.3970 for CsLAR $a$ and 0.7816 and 0.6474 for $C s L A R b$, respectively. The accumulation of the six catechins was negatively correlated with the expression of CsLARC.

\section{Functional verification of recombinant CsLARs expressed in E. coli}

Leucoanthocyanins, which are the direct substrates of LARs, are unstable and not commercially available. Therefore, we cannot directly detect the catalytic activity of the CsLAR proteins alone. Thus, we used a strategy that included coexpressing CsDFRa (KY615690) and LARs in E. coli to verify the function of the CsLARs with DHQ, DHK and DHM as the substrates (Fig. 4a). CsDFRa can catalyze these substrates to produce leucoanthocyanins, which then serve as the substrates for the CsLARs. The results of the enzymatic assay showed that CsDFRa coupled with CsLARc could catalyze DHQ to produce the product catechin. No product was detected with the empty vector, DFRa alone or the boiled DFRa + CsLARs controls, which indicated that our strategy was feasible (Fig. 4b). The three CsLARs combined with DFRa could catalyze the dihydroflavonols (DHK, DHQ and DHM) and generate the corresponding (2R, 3S)-trans-flavan-3-ols (AFZ, C and GC), which were identified using UPLC coupled to a triple quadrupole mass spectrometer (QQQ-MS) (Suppl. Fig. S3).

\section{Functional verification of the CsLARs in Arabidopsis}

In Arabidopsis thaliana, abundant PAs are present in the brown seed coat, including soluble and insoluble PAs, which mainly consist of an EC unit (Dixon et al. 2005). In addition, there is no LAR ortholog in the A. thaliana genome. 
Fig. 4 Identification of products from recombinant CsLARs in E. coli. a pRSF plasmid construct used for the co-expression of CsLARs and CsDFRa. b HPLC analysis of the product from the reaction of the recombinant proteins. Lines 1, 2, 3, 4 and 5 indicate the standards, empty vector control, CsDFRa alone reaction control, boiled enzyme control and CsDFRa + CsLARc assay, respectively a

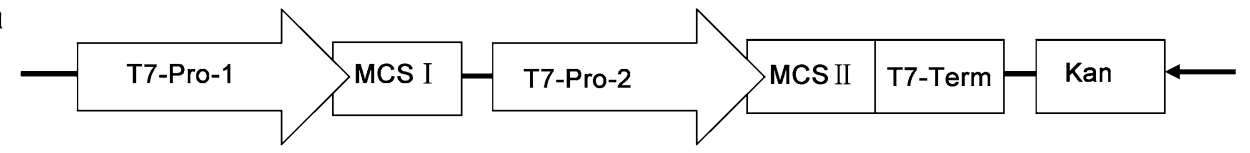

b

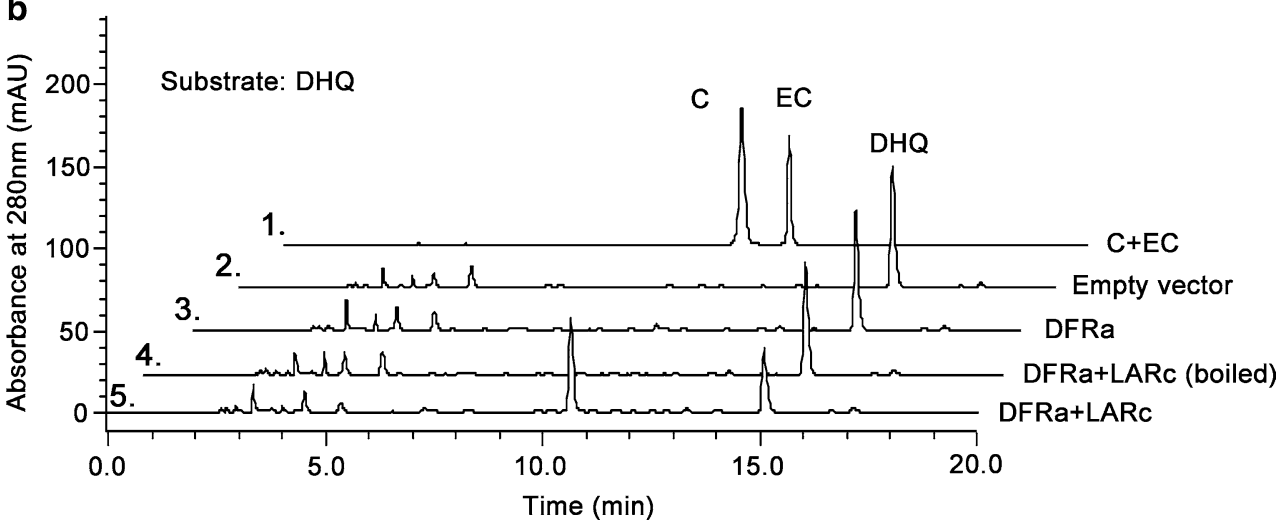

Therefore, A. thaliana is a suitable model plant to use to verify whether LAR is involved in PAs biosynthesis.

According to the phenotype of the transgenic A. thaliana overexpressing CsLARs, some lines showed a slight decrease in the brown color of the seed coat (data not shown). To detect the contents of the insoluble and soluble PAs in the seed coats, DMACA staining and an acid hydrolysis assay were conducted. The results showed that the contents of both insoluble and soluble PAs extracted from the seeds were reduced in the overexpressing CsLARs lines compared with WT (Fig. 5a, b). The UPLC-MRM analysis of the extracts showed that the contents of the ECmonomer $(\mathrm{m} / \mathrm{z} 289.0)$, PA-dimer $(\mathrm{m} / \mathrm{z}, 577.0)$ and PA-trimer ( $\mathrm{m} / \mathrm{z}$ 865.0) were decreased in the A. thaliana overexpressing CsLARs (Fig. 5c). This reduction in the EC monomers and polymers was consistent with the observed decrease in the brown color of the seed coat. Although CsLARs catalyzed leucocyanidins to produce catechin in vitro, no catechin could be detected in any transgenic $A$. thaliana lines, which was inconsistent with the results of the recombinant CsLARs in E. coli.

Dixon and coworkers found that MtLAR could catalyze a PA extension unit, $4 \beta$-( $S$-cysteinyl)-epicatechin, to form EC, resulting in a decrease of PAs (Liu et al. 2016). The degree of decrease in the EC-monomer was less than that of the polymers in the A. thaliana overexpressing CsLARs $(\mathrm{m} / \mathrm{z} 577.0, \mathrm{~m} / \mathrm{z}$ 865.0), which could be explained by this theory (Fig. 5c).

\section{Functional verification of the CsLARs in tobacco}

In contrast to A. thaliana, anthocyanins accumulate in the flowers of wild tobacco, and a slight accumulation of PAs was also detected due to the extremely low expression levels of the ANR and LAR genes. Thus, tobacco is a good model plant for studying the accumulation of anthocyanins and the biosynthesis of PAs.

To experimentally demonstrate the functions of the CsLARs, the open reading frames of the CsLARs were transferred into tobacco under the control of the Cauliflower mosaic virus $35 \mathrm{~S}$ promoter. More than 30 transgenic T1 lines of CsLARa, CsLARb and CsLARc were generated for further research. The petals of flowers overexpressing CsLARs showed a lighter pink color compared to the controls (empty vector, CK). In addition, the intensity of the pink color was negatively correlated with the transcriptional abundance of the CsLARs (Fig. 6b). Among these lines, certain CsLARc transgenic lines with high transcription levels became almost white (Fig. 6a). The extracts of the transgenic tobacco flowers exhibited blue staining after reacting with the DMACA reagent, indicating that DMACA-reactive compounds were generated in the tobacco. The content of the DMACA-reactive compounds in the flower petals increased, and the content of anthocyanins decreased as the expression levels of the CsLARs increased (Fig. 6c, d).

To determine whether there were oligomeric/polymeric PAs in the extracts and the petal residues, a butanol/ $\mathrm{HCl}$ hydrolysis assay was performed. No anthocyanin class material was generated after hydrolysis, indicating that there were no PAs in the transgenic tobacco (Suppl. Fig. S4). The content of the anthocyanin class material in the transgenic tobacco was lower than that in the control after the butanol/ $\mathrm{HCl}$ hydrolysis, indicating that overexpression of the CsLARs caused a decrease in the PAs.

UPLC-MS/MS analysis showed that the DMACAreactive compounds were the flavan-3-ol monomers EC and $\mathrm{C}$ (Fig. 6e). In addition, an EC (or C)-glycoside was also detected (Fig. 6f). The contents of EC, C and glycoside were positively correlated with the transcriptional abundance of the CsLARs (Fig. 6g). UPLC-MRM-MS 
Fig. 5 Overexpression of CsLARs in wild-type $A$. thaliana. a Soluble PAs content in WT and transgenic $A$. thaliana seeds. b Insoluble PAs content in WT and transgenic $A$. thaliana seeds. c The relative contents of EC, PA-dimers and PAtrimers in WT and transgenic A. thaliana seeds. The relative content is converted according to the peak area in a quantitative MRM model by UPLC-MS. All data are the means of three biological replicates, and the error bars represent the standard deviation of three replicates.

The contents of EC, PA-dimers and PA-trimers from WT were set as 1.0. The asterisks indicate the significant level $(n=3$, $* P<0.05, * * P<0.01)$ based on a Tukey's honestly significant difference test
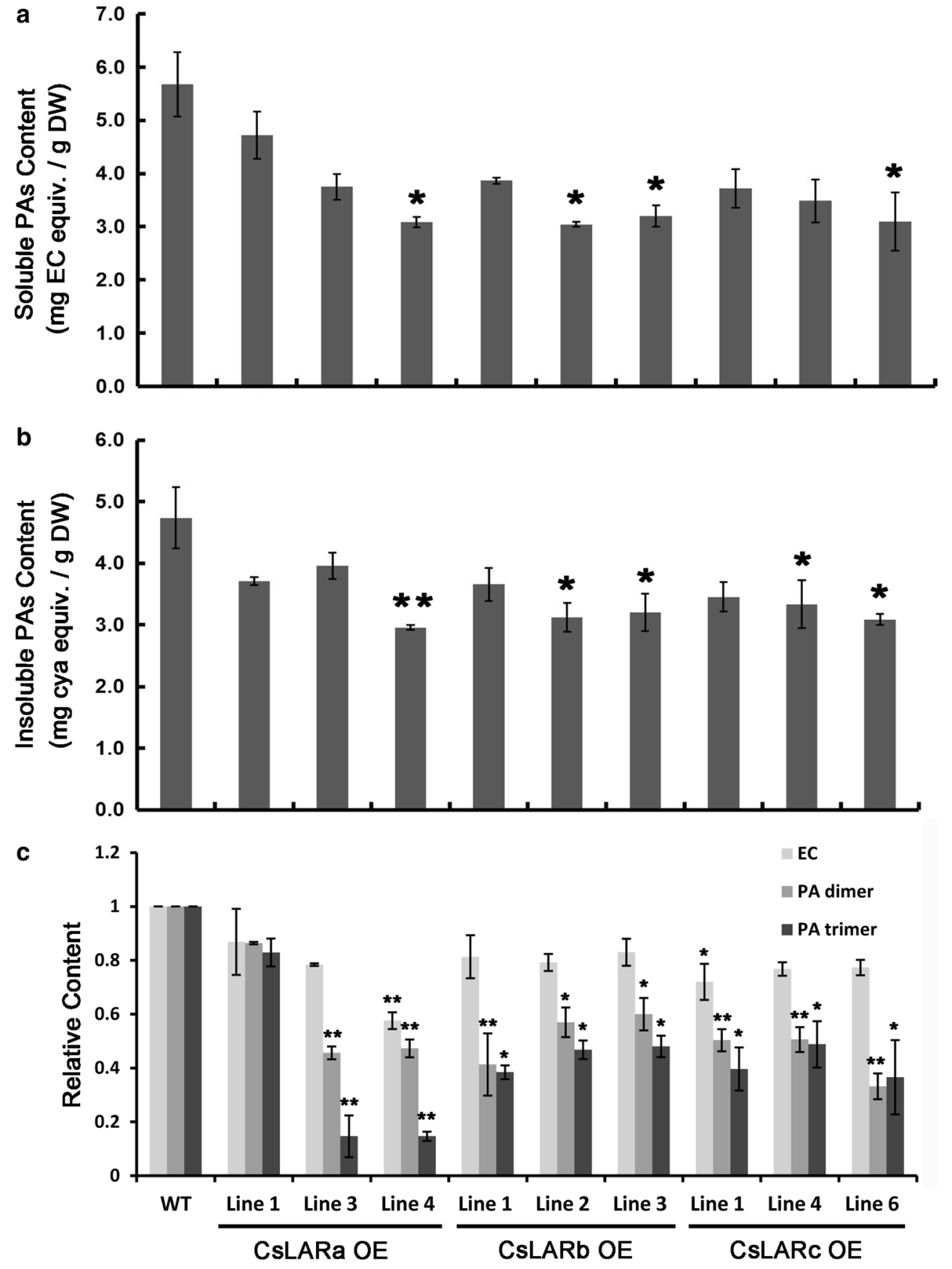

results showed that the accumulation of EC, C and glycoside in the CsLARc line was 8.58, 6.42 and 6.89 times greater than that in the control, respectively (Table 1).

In summary, the transgenic CsLARs tobacco flowers showed a visibly decreased color and produced a series of DMACA-reactive flavan-3-ol monomers containing mainly EC, C and glycoside. However, no PA oligomer/ polymer was detected in the CsLAR transgenic tobacco flowers.

\section{The interrelationships between the LAR and anthocyanidin pathways}

To determine the interrelationships between the LAR and anthocyanidin pathways, a hybridization experiment was performed using CsLARc and AtPAP1 transgenic tobacco that highly accumulated anthocyanins in the leaves and flowers. Through cross-pollination hybridization, several herbicide-resistant CsLARc + AtPAP1 transgenic tobacco 

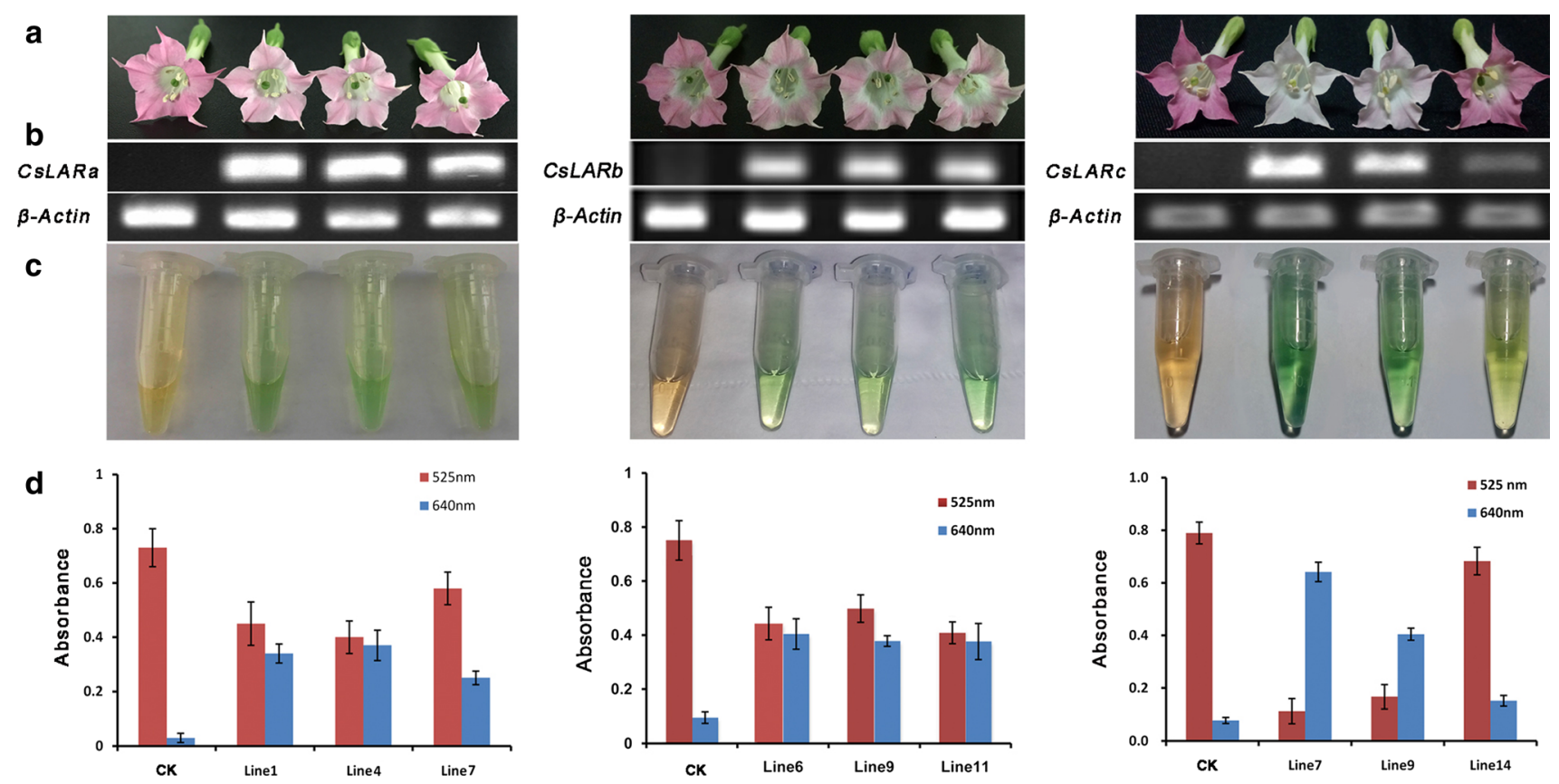

e

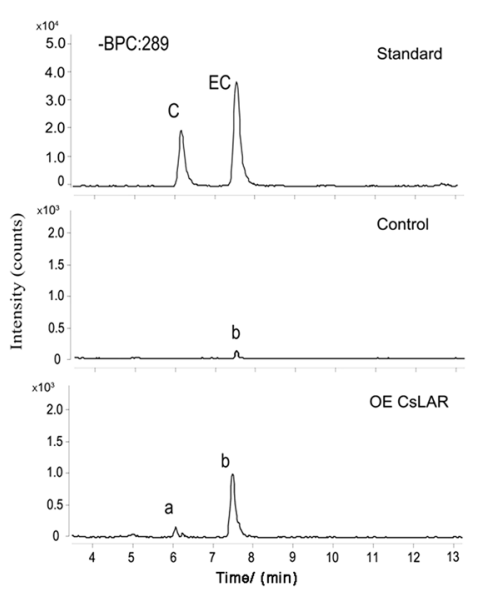

f

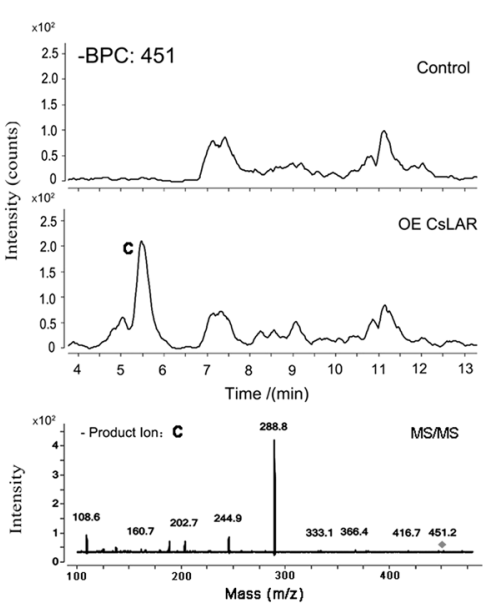

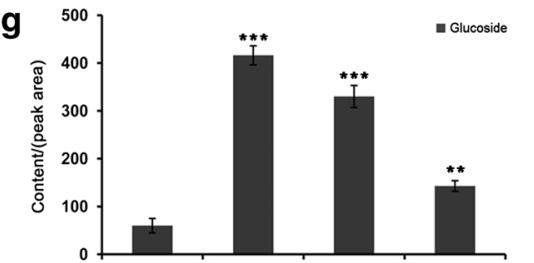

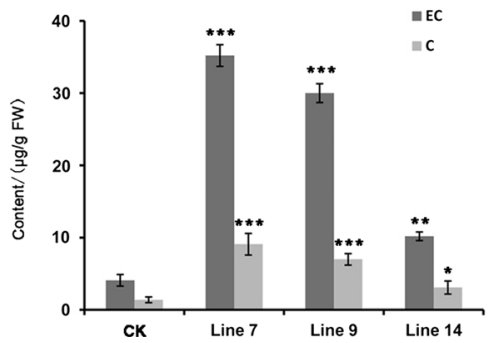

Fig. 6 Overexpression of CsLARs in wild-type tobacco. a Phenotypes of empty vector control (CK) and CsLARs transgenic tobacco flowers. b Semi-quantitative RT-PCR analysis of the CsLAR and the housekeeping gene NtActin transcription levels in total RNA from the flowers. c DMACA staining of the extracts from transgenic tobacco flowers and CK. d The absorbance of the anthocyanins extracts and DMACA-reactive compounds at 525 and $640 \mathrm{~nm}$, respectively. e Extraction ion chromatogram of products $(\mathrm{m} / \mathrm{z}, 289.0)$ accumulated in the transgenic tobacco flowers and CK. Authentic standard of catechin and epicatechin (top); products accumulated in the transgenic tobacco flowers (bottom) and CK (middle). f Extraction ion chromatogram of products $(\mathrm{m} / \mathrm{z}, 451.0)$ in the transgenic tobacco flowers (bot-

lines were generated. The transgenic lines with high transcription levels of both CsLARc and AtPAPI were selected for further analysis along with AtPAP1, CsLARc and CK tobacco lines as controls. Regarding the phenotype, the CsLARc + PAP1 transgenic tobacco exhibited a visibly reduced pink pigmentation in the leaves, stems and flowers tom) and CK (top). UPLC-MS/MS of peak c $(\mathrm{m} / \mathrm{z}$ 451.0) is listed in the box. $\mathrm{g}$ The contents of glycoside, $\mathrm{C}$ and EC extracted from different transgenic lines of overexpressing CsLARc tobacco flowers and CK. The content of glycoside is indicated by the peak area as determined by UPLC-MRM-MS. The contents (mg/g, FW) of C and EC were quantified according to the peak area based on the standard C and EC. All data are the means of three biological replicates, and the error bars represent the standard deviation of three replicates. The asterisks indicate the significance level $(n=3, * P<0.05$, $* * P<0.01, * * * P<0.001)$ based on a Tukey's honestly significant difference test

compared with the AtPAP1 controls, but the pigmentation was still darker than that in the CsLARc-expressing and CK tobacco lines (Fig. 7a, b). The measurement of the anthocyanins extracted from the flowers at $525 \mathrm{~nm}$ was consistent with the observed phenotype (Fig. 7c). DMACA-reactive compounds were generated only in the CsLARc + AtPAP1 
Table 1 Levels of selected flavonoid compounds in various transgenic and control tobacco. The data were determined by UPLC-MS. The content $(\mathrm{mg} / \mathrm{g}, \mathrm{FW})$ of $\mathrm{C}, \mathrm{EC}$ and procyanidin $\mathrm{B} 2$ were quanti-

\begin{tabular}{llccc}
\hline Compound name & \multicolumn{2}{l}{ Levels $(\mu \mathrm{g} / \mathrm{g}, \mathrm{FW})$} & & \\
\cline { 2 - 5 } & CK $($ control $)$ & CsLARc & CsLARc + AtPAP1 & AtPAP1 \\
\hline EC $(\mathrm{C})$-glycoside* & 60.3 & 415.6 & 1615.5 & 38.08 \\
Catechin & 1.19 & 7.64 & 128.72 & 1.46 \\
Epicatechin & 3.47 & 29.79 & 0.88 & 4.74 \\
PA-dimers (B2) & 0.74 & 0.32 & 10.52 & 1.09 \\
PA-trimers* & 20.31 & 8.92 & 25.1 \\
\hline
\end{tabular}

The asterisk represents the substance for which we do not have the standards. So, the values represent the peak area

and CsLARc transgenic tobacco, and the absorbance value of CsLARc + PAP1 was double that of the CsLARc transgenic tobacco. The UPLC-MRM-MS analysis indicated that the accumulation of EC, C and glycoside in the CsLARc + AtPAP1 line was 4.32, 4.98 and 3.88 times greater than that in the CsLARc line and 27.15, 26.08 and 24.70 times greater than that in the AtPAP1 line, respectively (Table 1). The contents of the PA-dimers and -trimers in the flower extracts of the CsLARc + AtPAP1 line were 0.80 and 0.42 -fold greater than those in the AtPAP1 line, respectively. Similarly, no insoluble PAs were detected in the residues of any transgenic lines by the butanol/ $\mathrm{HCl}$ hydrolysis assay. Overexpression of the LARs reduced the content of anthocyanins and accumulated EC, C and glycoside in the tobacco, but it did not promote the biosynthesis of PAs.

\section{The coefficients of LAR and ANR in the PA biosynthetic pathway}

Certain R2R3 MYB transcription factors, such as AtTT2, VvMYBPA1 and MtMYB14, have been reported to regulate the biosynthesis of PAs by regulating $A N R$ and $L A R$ (Nesi et al. 2001; Bogs et al. 2007; Liu et al. 2014). According to the conserved amino acid sequence, a homologous TT2 R2R3 MYB transcription factor, named CsMYB5b (KY827397), in the tea plant was selected and overexpressed in tobacco to examine the interrelationships between LAR and ANR.

The phenotypes of transgenic CsMYB5b-expressing tobacco were consistent with those of transgenic CsLAR tobacco (Fig. 8a). The petals of flowers overexpressing CsMYB5b also exhibited lighter pink color, and the intensity of the pink color was negatively correlated with the transcription levels of CsMYB5b. Simultaneously, the content of the DMACA-reactive compounds was increased with the increase in the transcription levels of $C S M Y B 5 b$ (Fig. $8 \mathrm{~b}-\mathrm{d}$ ).

Unlike the CsLARs transgenic tobacco, EC, C and glucoside were detected, but additionally, PA polymers $(\mathrm{m} / \mathrm{z}$ 577.0 and $m / z$ 865.0) accumulated in the overexpressing fied according to the peak area based on the standards. All data are the mean values of three biological replicates
CsMYB5b tobacco, and their accumulation was positively correlated with the expression levels of $C s M Y B 5 b$ (Fig. 8e, f). Among them, the content of EC was three times greater than $\mathrm{C}$, and the PA-dimer was ten times greater than the PA-trimer. Butanol/ $\mathrm{HCl}$ hydrolysis experiments also showed that the overexpressing CsMYB5b tobacco accumulated a massive amount of PA polymers (including soluble and insoluble PAs), which were never detected in the tobacco overexpressing CsLARs (Suppl. Fig. S3). qRT-PCR analysis revealed that $N t A N R 1, N t A N R 2$ and $N t L A R$ were the main up-regulated genes in all of the transgenic lines (Fig. $8 \mathrm{~g}$ ). $N t 4 C L, N t C 4 H, N t F 3^{\prime} H$ and $N t F L S$, which are involved in the flavonoid pathway, were up-regulated (more than twofold) in CsMYB5b transgenic tobacco. Unlike NtANR1, NtANR2 and NtLAR, NtC4H, NtDFR and NtANS only exhibited higher expression levels in transgenic Line 1 (Fig. 8g). The results implied that the biosynthesis of PAs might require the co-expression of NtANRs and NtLAR. Expression of LAR alone only promoted the biosynthesis of flavan3-ol monomers. The primers used in qRT-PCR are listed in Suppl. Table S2.

\section{Discussion}

\section{Evolutionary analysis of LARs in plants}

Flavonoids evolution has been well discussed from the phylogenetic, chemotaxonomic, enzymatic, and molecular perspectives (Harborne 1989; Stafford 1991; Koes et al. 1994; Grotewold 2006). The enormous variation in the types of flavonoids produced by different plants may be due to gene duplication and divergence, pleiotropy and loss-of-function mutations that occurred during adaptive evolutionary changes (Grotewold 2006). For example, the emergence of certain new enzymes or functions resulted from initial gene duplication, such as $\mathrm{CHS}, \mathrm{F3H}$ and $\mathrm{CHI}$ (Verwoert et al. 1992; Jez et al. 2000; Winkel-Shirley 2001). The biosynthetic branch pathway of PAs can be traced back to ferns, 
a

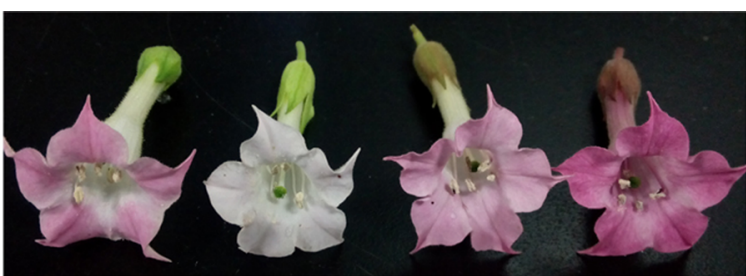

b

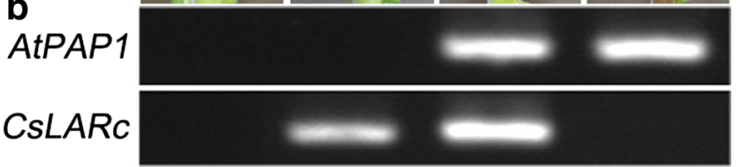

C

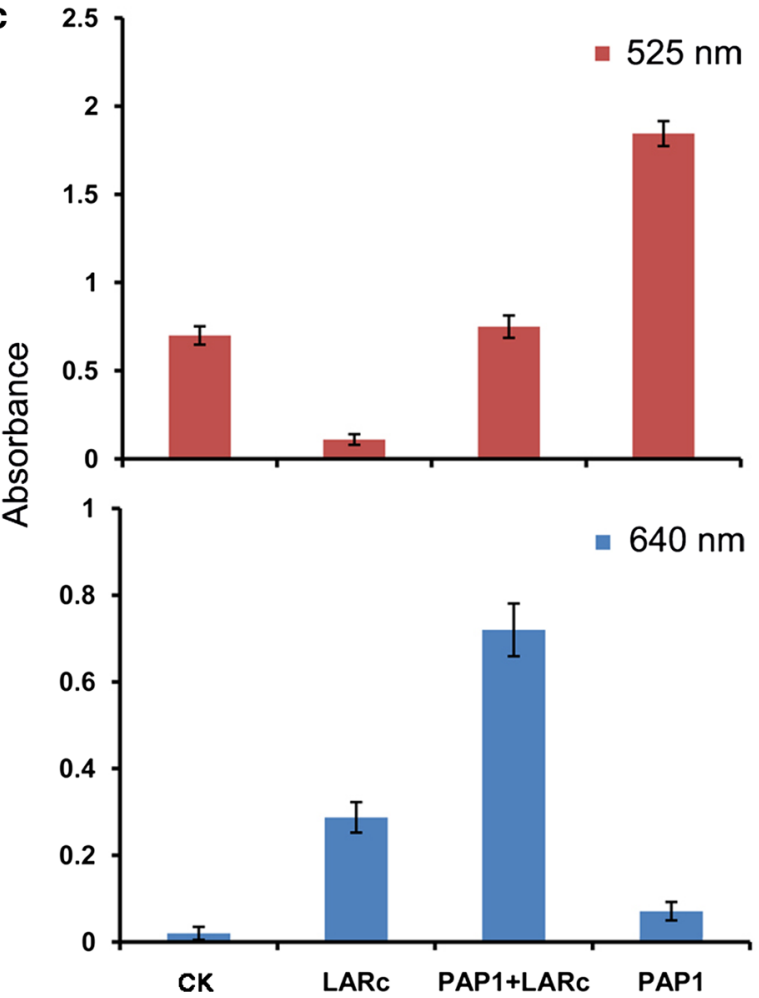

Fig. 7 Hybridization of the CsLARc transgenic tobacco and AtPAP1 transgenic tobacco. a Phenotypes of CK, CsLARc, AtPAP1 and CsLARc + AtPAP1 transgenic tobacco. b Semi-quantitative RTPCR analysis of the CsLARc and AtPAP1 transcription levels in total RNA from the flowers shown in a. $\mathbf{c}$ The absorbance of the anthocyanins extracts and DMACA-reactive compounds at 525 and $640 \mathrm{~nm}$, respectively

which are the most primitive vascular plants (Grotewold 2006). The flavanols, which are precursors of PA biosynthesis, are widely distributed in gymnosperms and angiosperms and have also been detected in ferns (Stafford 1991; Peng et al. 2012). Many experiments have shown that the accumulation of phenolic compounds is closely related to plant resistance against biotic and abiotic stresses, which contributes to the adaptation of plants to environmental stresses (Dixon et al. 2005; Fornalé et al. 2014). An independent evolution of a specific F3'5'H in the Asteraceae family, which provides the basis for their purple flower color, likely reflects the adaptive value of attraction for insect pollination (Seitz et al. 2006).

The LARs and ANRs are known to be responsible for the biosynthesis of flavan-3-ols (Tanner et al. 2003; Xie et al. 2004). As shown in the phylogenetic tree (Fig. 3), the LARs are widely distributed from gymnosperms to angiosperms, although an LAR homolog has not been found in ferns. This finding is consistent with the evolutionary analysis of the ANR homologs, which are present in gymnosperms and dicotyledonous and monocotyledonous species (Peng et al. 2012).

The dicotyledonous LARs could be further clustered into two subgroups, i.e., cluster I and cluster II. Nevertheless, the LARs from Leguminosae, such as G. max, L. corniculatus, $P$. sativum and $M$. truncatula, constituted a monophyletic group that belonged to cluster I. Similarly, a previously reported phylogenetic tree of the $D F R$ genes also showed that the DFRs of leguminous plants constituted a monophyletic group (Shimada et al. 2005). The evolutionary significance of this phenomenon is currently unclear.

LARs in plants were classified as G-type LARs (gymnosperms and monocotyledons groups), A-type LARs (dicotyledonous cluster II) and S-type LARs (dicotyledonous cluster I) based on a variable amino acid in the conserved ICCN motif. Further analysis revealed that a transversion $(\mathrm{G} \rightarrow \mathrm{T})$ and a transition $(\mathrm{G} \rightarrow \mathrm{C})$ mutation occurred in the first and second positions of this codon, respectively. Though the ICCN motif is located near the substrate-binding site according to VvLAR protein crystal structure, we did not see an obvious difference among the three CsLARs (CsLARa, b belong to S-type LARs and CsLARc belongs to A-type LARs) in either in vivo or in vitro experiments. A previously reported PaLAR3 (a G-type LAR, from gymnosperm Picea abies) also had the same function of catalyzing leucocyanidin to produce catechin in vitro (Hammerbacher et al. 2014). It suggests that the effect of this polymorphism is likely to be quite subtle.

Protein evolution has been studied for many years (Echave and Wilke 2016). Studying whether the mutation in the eighth amino acid in the ICCN motif affects the protein function, evolutionary rate, kinetic stability, native state stability and active structure stability of the LAR protein is warranted.

\section{The predicted function of LAR in plants}

To date, all LARs in different plants have been reported to have the catalytic products AFZ, C and GC in vitro with 
Fig. 8 Overexpression of CsMYB5b in wild-type tobacco. a Phenotypes of CK and CsMYB5b transgenic tobacco flowers. b Semi-quantitative RT-PCR analysis of the $C s M Y B 5 b$ and the housekeeping gene NtActin transcription levels in total RNA from the flowers shown in a. c DMACA staining of the extracts from transgenic tobacco flowers and controls. d The absorbance of the anthocyanins extracts and DMACA-reactive compounds at 525 and $640 \mathrm{~nm}$, respectively. e The content (peak area) of glycosides extracted from different transgenic lines of overexpressing CsMYB5b tobacco flowers and CK. f The contents $(\mu \mathrm{g} / \mathrm{g}$, FW) of C, EC, PA-dimer and PA-trimer extracted from different transgenic lines of overexpressing CsMYB5b tobacco flowers and CK. The PA-trimer was also quantified according to the standard procyanidin $\mathrm{B} 2$. All data are the means of three biological replicates, and the error bars represent the standard deviation of three replicates. The asterisks indicate the significant level $(n=3, * P<0.05$, $* * P<0.01, * * * P<0.001)$ based on a Tukey's honestly significant difference test. $\mathbf{g}$ Expression profiles of structural genes in flowers of different transgenic CsMYB5b tobacco lines
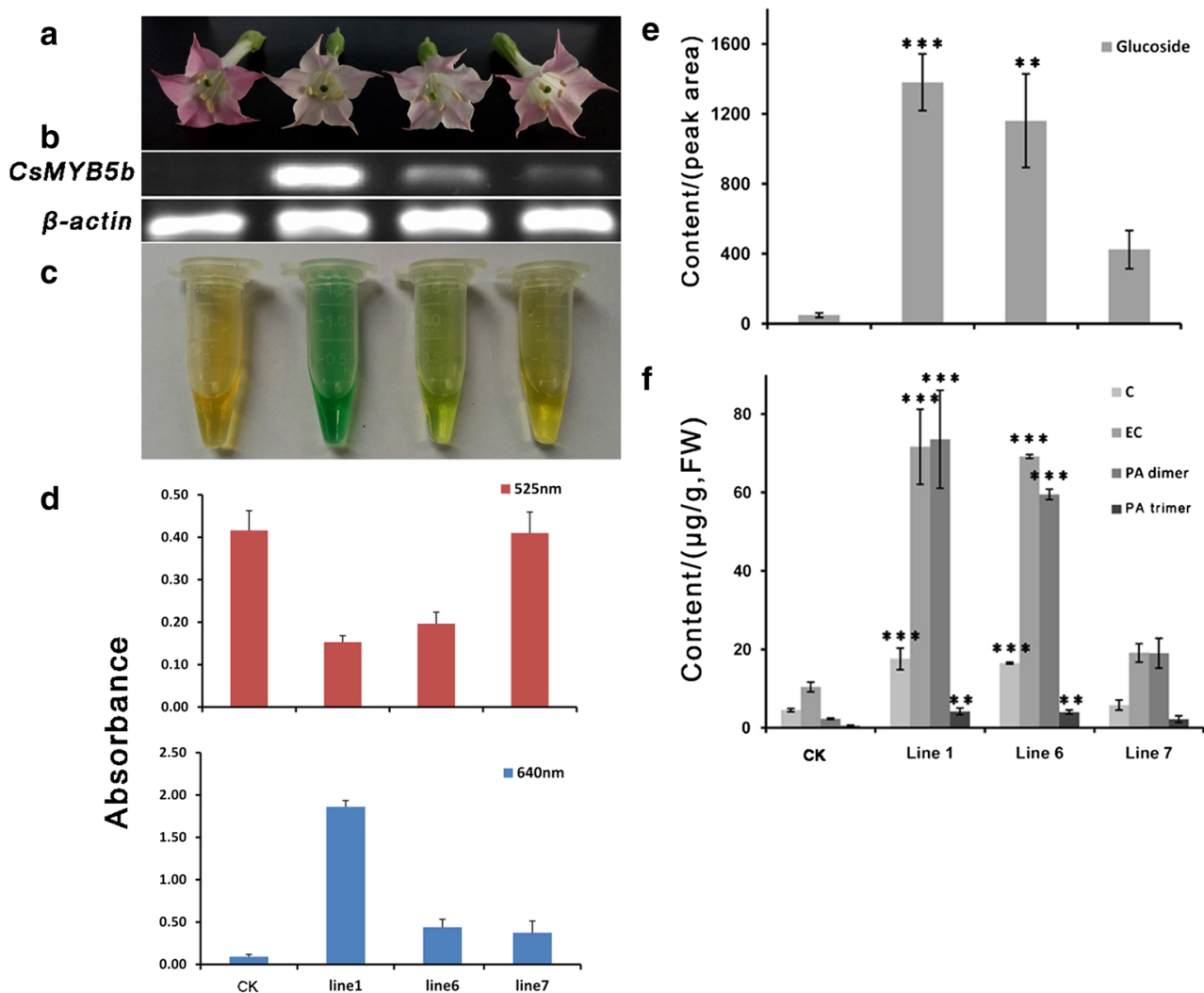

\section{f}

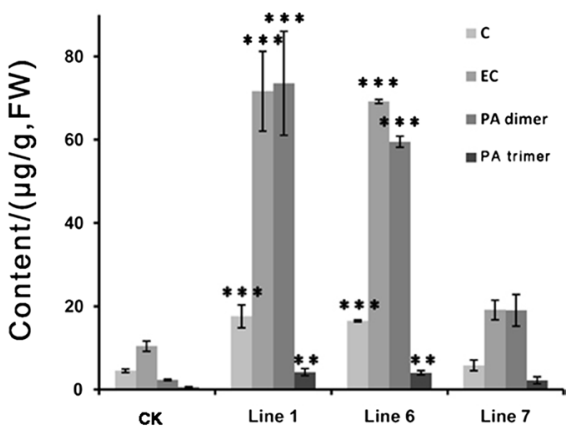

g

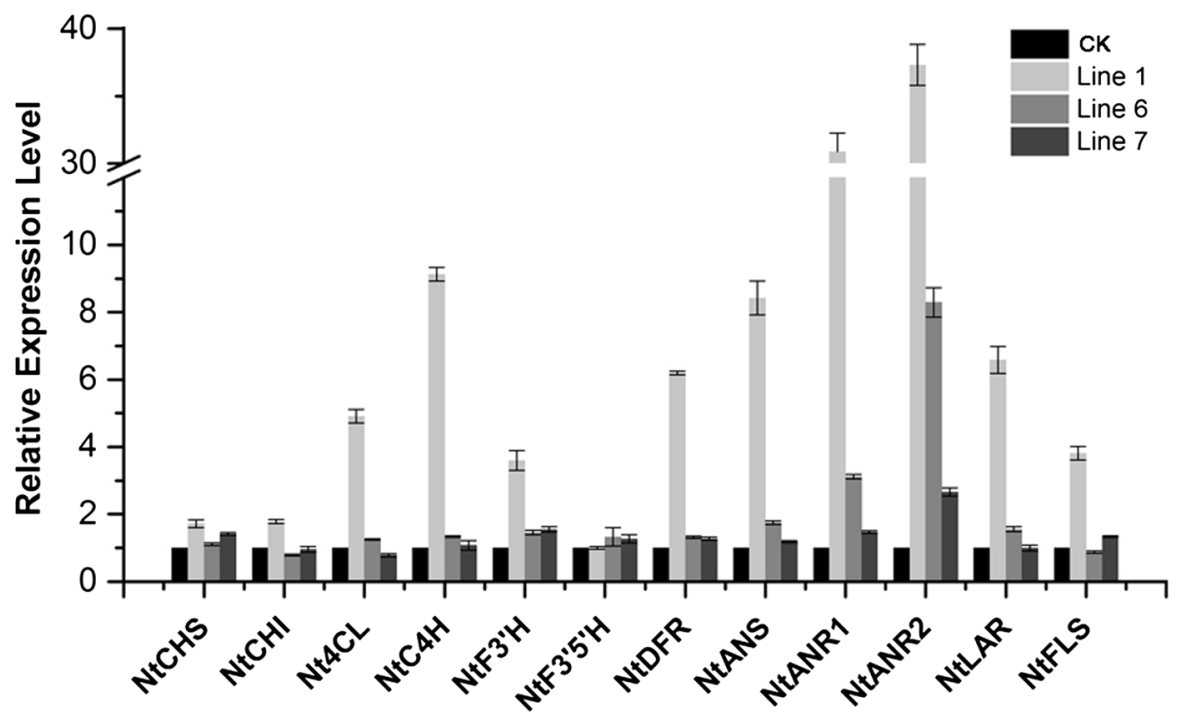

leucoanthocyanidins as substrates (Stafford and Lester 1984; Kristiansen 1986; Tanner et al. 2003; Pang et al. 2007). However, transgenic plants constitutively overexpressing LARs resulted in different characteristics, which raised new questions regarding the real function of LARs in vivo. In transgenic DuLAR tobacco and white clover plants, no detectable levels of catechin or polymer were found (Tanner et al. 2003). The overexpression of MtLAR in tobacco reduced the content of anthocyanins in the flower petals, but no desired catechin or increased PAs were detected in the flowers or leaves (Pang et al. 2007). The transgenic TcLAR tobacco showed decreased amount of anthocyanidins and unexpectedly generated both EC and $\mathrm{C}$ monomers in the tobacco flowers (Liu et al. 2013). The ectopic expression of CsLAR in the tea cultivar TRI2043 in M. truncatula hairy roots and a type of purple tobacco accumulated a massive amount of anthocyanins and EC, respectively (Pang et al. 2013). In our experiments, the coupled DFR + LAR assays indicated that a single product of $\mathrm{C}$ was generated and DHQ served as the substrate. The overexpression of CsLARs in 
tobacco and the hybridization assay of CsLARc and AtPAP1 both indicated that $\mathrm{EC}$ was the main product, along with the synthesis of minus $\mathrm{C}$. In addition, no increased polymers were detected in the extracts or residues. In contrast, the content of the PAs in the tobacco overexpressing CsLARs was reduced (Suppl. Fig. S3). This finding is somewhat consistent with the findings of a decrease in the PAs content in flowers from transgenic tobacco overexpressing MdLAR1 or an increase in the insoluble PAs in the seeds of the lar M. truncatula mutants (Liao et al. 2014; Liu et al. 2016). Therefore, we hypothesize that other substrates of LAR may exist in tobacco in addition to leucocyanidins.

This supposition has recently been explored. Dixon and coworkers found that MtLAR could catalyze a new substrate, $4 \beta$-( $S$-cysteinyl)-epicatechin, to form EC in vitro (Liu et al. 2016). Excessive $4 \beta$-( $S$-cysteinyl)-epicatechin, which is a carbocation form, played an important role in non-enzymatic polymerization and served as a PA extension unit in $M$. truncatula. Yet, the source of $4 \beta$-( $S$-cysteinyl)-epicatechin in M. truncatula is unknown. The biosynthesis of carbocation is particularly important, and a further understanding of this process will be helpful for more rationally explaining the true function of LAR in vivo. In addition, more direct genetic evidence, such as mutations in LAR, need to be obtained to elucidate the real function of LAR in vivo.

Author contribution statement LG and TX conceived and designed the experiment. PW performed most of experiments and wrote the paper. LZ and TL cultivated the transgenic tobacco. XJ analyzed the data of LC-MS. $\mathrm{XD}$ extracted and determined the metabolites of transgenic tobacco. LX and DX extracted and determined the metabolites of transgenic Arabidopsis thaliana. YL and ML modified the language of the paper. All authors read and approved the final version of the manuscript.

Acknowledgements This work was financially supported by the Natural Science Foundation of China (31470689, 31270730, 31200229 and 31570694). The authors are grateful to the reviewers for their insightful suggestions and comments.

\section{Compliance with ethical standards}

Conflict of interest The authors declare that they have no conflicts of interest with the contents of this article.

Open Access This article is distributed under the terms of the Creative Commons Attribution 4.0 International License (http://creativecommons.org/licenses/by/4.0/), which permits unrestricted use, distribution, and reproduction in any medium, provided you give appropriate credit to the original author(s) and the source, provide a link to the Creative Commons license, and indicate if changes were made.

\section{References}

Bogs J, Downey MO, Harvey JS, Ashton AR, Tanner GJ, Robinson SP (2005) Proanthocyanidin synthesis and expression of genes encoding leucoanthocyanidin reductase and anthocyanidin reductase in developing grape berries and grapevine leaves. Plant Physiol 139:652-663

Bogs J, Jaffé FW, Takos AM, Walker AR, Robinson SP (2007) The grapevine transcription factor VvMYBPA1 regulates proanthocyanidin synthesis during fruit development. Plant Physiol 143:1347-1361

Cai XT, Xu P, Zhao PX, Liu R, Yu LH, Xiang CB (2014) Arabidopsis ERF109 mediates cross-talk between jasmonic acid and auxin biosynthesis during lateral root formation. Nat Commun 5:5833

Chung FL, Schwartz J, Herzog CR, Yang YM (2003) Tea and cancer prevention: studies in animals and humans. J Nutr 133:3268S-3274S

Clough SJ, Bent AF (1998) Floral dip: a simplified method for Agrobacterium-mediated transformation of Arabidopsis thaliana. Plant J 16:735-743

Cos P, De BT, Hermans N, Apers S, Berghe DV, Vlietinck AJ (2004) Proanthocyanidins in health care: current and new trends. Curr Med Chem 11:1345-1359

Cui LL, Yao SB, Dai XL et al (2016) Identification of UDP-glycosyltransferases involved in the biosynthesis of astringent taste compounds in tea (Camellia sinensis). J Exp Bot 67:2285-2297

Dixon RA, Sumner LW (2003) Legume natural products: understanding and manipulating complex pathways for human and animal health. Plant Physiol 131:878-885

Dixon RA, Xie DY, Sharma SB (2005) Proanthocyanidins-a final frontier in flavonoid research? New Phytol 165:9-28

Echave J, Wilke CO (2016) Biophysical models of protein evolution: understanding the patterns of evolutionary sequence divergence. Annu Rev Biophys 46:85-103

Fisher DK, Guiltinan MJ (1995) Rapid, efficient production of homozygous transgenic tobacco plants with agrobacterium tumefaciens: a seed-to-seed protocol. Plant Mol Biol Rep 13:278-289

Fornalé S, Lopez E, Salazarhenao JE, Fernándeznohales P, Rigau J, Caparrosruiz D (2014) AtMYB7, a new player in the regulation of UV-sunscreens in Arabidopsis thaliana. Plant Cell Physiol $55: 507-516$

Grotewold E (2006) The science of flavonoids. Springer, New York

Hammerbacher A, Paetz C, Wright LP, Fischer TC, Bohlmann J, Davis AJ, Fenning TM, Gershenzon J, Schmidt A (2014) Flavan-3-ols in Norway spruce: biosynthesis, accumulation, and function in response to attack by the bark beetle-associated fungus Ceratocystis polonica. Plant Physiol 164:2107-2122

Harborne JB (ed) (1989) The flavonoids-advances in research since 1980. Chapman \& Hall, London

Jez JM, Bowman ME, Dixon RA, Noel JP (2000) Structure and mechanism of the evolutionarily unique plant enzyme chalcone isomerase. Nat Struct Biol 7:786

Jiang XL, Liu YJ, Li WW, Zhao L, Meng F, Wang YS, Tan HR, Yang H, Wei CL, Wan XC, Gao LP, Xia T (2013) Tissue-specific, development-dependent phenolic compounds accumulation profile and gene expression pattern in tea plant (Camellia sinensis). PLoS One 8:e62315-e62315

Jiang XL, Liu YJ, Wu YH, Tan HR, Meng F, Wang YS, Li MZ, Zhao L, Liu L, Qian YM, Gao LP, Xia T (2015) Analysis of accumulation patterns and preliminary study on the condensation mechanism of proanthocyanidins in the tea plant (Camellia sinensis). Sci Rep $5: 8742$ 
Kennedy JA, Ferrier J, Harbertson JF, Peyrot dGC (2008) Analysis of tannins in red wine using multiple methods: correlation with perceived astringency. Am J Enol Vitic 57:481-485

Koes RE, Quattrocchio F, Mol JNM (1994) The flavonoid biosynthetic pathway in plants: function and evolution. BioEssays 16:123-132

Kristiansen KN (1986) Conversion of (+)-dihydroquercetin to (+)-2,3-trans-3,4-cis-leucocyanidin and (+)-catechin with an enzyme extract from maturing grains of barley. Carlsberg Res Commun 51:51-60

Li YG, Tanner G, Larkin P (1996) The DMACA-HCl protocol and the threshold proanthocyanidin content for bloat safety in forage legumes. J Sci Food Agric 70:89-101

Li Y, Wang L, Han Z, Jiang Y, Zhao L, Hong L, Li Y, Luo K (2012) Molecular cloning and characterization of PtrLAR3, a gene encoding leucoanthocyanidin reductase from Populus trichocarpa, and its constitutive expression enhances fungal resistance in transgenic plants. J Exp Bot 63:2513-2524

Liao L, Vimolmangkang S, Wei G, Zhou H, Korban SS, Han Y (2014) Molecular characterization of genes encoding leucoanthocyanidin reductase involved in proanthocyanidin biosynthesis in apple. Front Plant Sci 6:243

Liu Y, Gao L, Liu L, Yang Q, Lu Z, Nie Z, Wang Y, Xia T (2012) Purification and characterization of a novel galloyltransferase involved in catechin galloylation in the tea plant (Camellia sinensis). J Biol Chem 287:44406-44417

Liu Y, Shi Z, Maximova S, Payne MJ, Guiltinan MJ (2013) Proanthocyanidin synthesis in Theobroma cacao: genes encoding anthocyanidin synthase, anthocyanidin reductase, and leucoanthocyanidin reductase. BMC Plant Biol 13:202

Liu C, Jun JH, Dixon RA (2014) MYB5 and MYB14 play pivotal roles in seed coat polymer biosynthesis in Medicago truncatula. Plant Physiol 165:1424

Liu C, Wang X, Shulaev V, Dixon RA (2016) A role for leucoanthocyanidin reductase in the extension of proanthocyanidins. Nat Plants 2:16182

Ma CM, Sato N, Li XY, Nakamura N, Hattori M (2010) Flavan-3-ol contents, anti-oxidative and $\alpha$-glucosidase inhibitory activities of Cynomorium songaricum. Food Chem 118:116-119

Mauge C, Granier Td'Estaintot BL, Gargouri M, Manigand C, Schmitter JM, Chaudiere J, Gallois B (2010) Crystal structure and catalytic mechanism of leucoanthocyanidin reductase from Vitis vinifera. J Mol Biol 397:1079-1091

Mellway RD, Lan TT, Prouse MB, Campbell MM, Constabel CP (2009) The wound-, pathogen-, and ultraviolet B-responsive MYB134 gene encodes an R2R3 MYB transcription factor that regulates proanthocyanidin synthesis in poplar. Plant Physiol 150:924-941

Nesi N, Jond C, Debeaujon I, Caboche M, Lepiniec L (2001) The Arabidopsis TT2 gene encodes an R2R3 MYB domain protein that acts as a key determinant for proanthocyanidin accumulation in developing seed. Plant Cell 13:2099-2114

Nichols JA, Katiyar SK (2010) Skin photoprotection by natural polyphenols: anti-inflammatory, antioxidant and DNA repair mechanisms. Arch Dermatol Res 302:71-83

Pang Y, Peel GJ, Wright E, Wang Z, Dixon RA (2007) Early steps in proanthocyanidin biosynthesis in the model legume Medicago truncatula. Plant Physiol 145:601-615
Pang Y, Abeysinghe ISB, He J, He XZ, Huhman D, Mewan KM, Sumner LW, Yun J, Dixon RA (2013) Functional characterization of proanthocyanidin pathway enzymes from tea and their application for metabolic engineering. Plant Physiol 161:1103

Peng QZ, Zhu Y, Liu Z, Du C, Li KG, Xie DY (2012) An integrated approach to demonstrating the ANR pathway of proanthocyanidin biosynthesis in plants. Planta 236:901-918

Qian YM, Zhao XQ, Zhao L, Cui LL, Liu L, Jiang XL, Liu YJ, Gao LP, Xia T (2015) Analysis of stereochemistry and biosynthesis of epicatechin in tea plants by chiral phase high performance liquid chromatography. J Chromatogr B Anal Technol Biomed Life Sci 1006:1-7

Seitz C, Eder C, Deiml B, Kellner S, Martens S, Forkmann G (2006) Cloning, functional identification and sequence analysis of flavonoid 3'-hydroxylase and flavonoid 3 ', $5^{\prime}$-hydroxylase cDNAs reveals independent evolution of flavonoid $3^{\prime}, 5^{\prime}$-hydroxylase in the Asteraceae family. Plant Mol Biol 61:365-381

Shimada N, Sasaki R, Sato S, Kaneko T, Tabata S, Aoki T, Ayabe S (2005) A comprehensive analysis of six dihydroflavonol 4-reductases encoded by a gene cluster of the Lotus japonicus genome. J Exp Bot 56:2573

Stafford HA (1991) Flavonoid evolution-an enzymatic approach. Plant Physiol 96:680-685

Stafford HA, Lester HH (1982) Enzymic and nonenzymic reduction of (+)-dihydroquercetin to its 3,4,-diol. Plant Physiol 70:695-698

Stafford HA, Lester HH (1984) Flavan-3-ol biosynthesis: the conversion of (+)-dihydroquercetin and flavan-3,4-cis-diol (leucocyanidin) to $(+)$-catechin by reductases extracted from cell suspension cultures of douglas fir. Plant Physiol 76:184-186

Tanner GJ, Francki KT, Abrahams S, Watson JM, Larkin PJ, Ashton AR (2003) Proanthocyanidin biosynthesis in plants. Purification of legume leucoanthocyanidin reductase and molecular cloning of its cDNA. J Biol Chem 278:31647-31656

Verwoert II, Verbree EC, Kh VDL, Nijkamp HJ, Stuitje AR (1992) Cloning, nucleotide sequence, and expression of the Escherichia coli fabD gene, encoding malonyl coenzyme A-acyl carrier protein transacylase. J Bacteriol 174:2851

Wang Y, Chun OK, Song WO (2013) Plasma and dietary antioxidant status as cardiovascular disease risk factors: a review of human studies. Nutrients 5:2969-3004

Wang YS, Xu YJ, Gao LP, Yu O, Wang XZ, He XJ, Jiang XL, Liu YJ, Xia T (2014) Functional analysis of flavonoid $3^{\prime}, 5^{\prime}$-hydroxylase from tea plant (Camellia sinensis): critical role in the accumulation of catechins. BMC Plant Biol 14:347

Winkel-Shirley B (2001) Flavonoid biosynthesis. A colorful model for genetics, biochemistry, cell biology, and biotechnology. Plant Physiol 126:485-493

Wu YH, Jiang XL, Zhang SX, Dai XL, Liu YJ, Tan H, Gao LP, Xia T (2016) Quantification of flavonol glycosides in Camellia sinensis by MRM mode of UPLC-QQQ-MS/MS. J Chromatogr B Anal Technol Biomed Life Sci 1017-1018:10

Xie DY, Sharma SB, Paiva NL, Ferreira D, Dixon RA (2003) Role of anthocyanidin reductase, encoded by BANYULS in plant flavonoid biosynthesis. Science 299:396-399

Xie DY, Sharma SB, Dixon RA (2004) Anthocyanidin reductases from Medicago truncatula and Arabidopsis thaliana. Arch Biochem Biophys 422:91-102 